\title{
Identification of Escherichia coli Genes Essential for High-Level, Clinically Relevant, Resistance to Antibiotics
}

\author{
By
}

Esmeralda Z. Reyes-Fernández ${ }^{1,2}$, Noemie Alon Cudkowicz ${ }^{1}$, Sonia Steiner-Mordoch ${ }^{1}$ and Shimon Schuldiner*

Dep. Biological Chemistry, Institute of Life Sciences, Silberman Bldg. 1-339, Edmond J. Safra

Campus, Hebrew University of Jerusalem, Givat Ram, Jerusalem 91904, Israel

Email: shimon.schuldiner@huji.ac.il

1. These authors contributed equally to the paper

2. Present address: Department of Microbiology and Immunology, Albert Einstein

College of Medicine, Bronx, NY

Keywords: Multiple Antibiotic

Resistance/transposon/quinolones/yhdP/yiho/waa/sulfoglucose transporter

Abbreviations: Antimicrobial Resistance (AMR), Minimal Medium A (MMA), Norfloxacin

(norfloxacin) 


\section{ABSTRACT}

Antibiotic resistance is one of the biggest public health challenges of our time. Here we present a novel approach to recognizing molecular mechanisms essential for maintaining high-level clinically relevant antibiotic resistance. To identify essential genes in this context, we subjected Escherichia coli EV18, a strain highly resistant to quinolones, to random transposon mutagenesis. This strain's unique advantage is that the screen is performed at very high concentrations of the antibiotic, non-permissive for most strains. The transposon's insertion affected the transcription of five genes required for the maintenance of resistance in EV18. Three of these genes (YihO, YhdP, and WaaY) have not been previously identified as essential for high-level antimicrobial resistance (AMR). Our work provides a new perspective to identify and characterize novel players crucial for maintaining AMR in E. coli.

\section{INTRODUCTION}

Antibiotic resistance poses a formidable problem in hospital-acquired infections. Many findings suggest that inadequate use of antimicrobials may lead to resistance in bacteria (AMR) and make the treatment of bacterial infections more difficult (1-4). Extensive knowledge of the molecular mechanisms underlying microbial antibiotic resistance is required to fight the increasing numbers of drug-resistant and multidrug-resistant bacteria successfully.

Many genes are involved in Escherichia coli and other organisms' response to antibiotic exposure and AMR acquisition (5). Resistance to antibiotics can be caused generally by the inactivation or modification of the antibiotic, alteration in the target site of the antibiotic, reduction of the intracellular antibiotic accumulation by increasing the active efflux of the antibiotic and modification of metabolic pathways to circumvent the antibiotic effect $(6-11)$.

In an in-lab evolution experiment, we identified Multidrug Transporters (MDTs)' that play a central role in the process of acquisition of high level clinically relevant resistance but, in this strain, they are not essential for maintenance (12). Therefore, E. coli EV18, our evolved strain, provides an excellent tool to identify genes essential for maintenance other than MDTs. 
Random mutagenesis has been considered one of the strategies to study genes' expression and function with crucial roles in different biological processes. Transposition based strategies have been used successfully to generate mutant transposon libraries, for site-specific tagging, and the generation of transcriptional/translational target gene fusions (13-18). To identify genes essential for maintaining high-level, clinically relevant antibiotic resistance, we subjected EV18, to random transposon mutagenesis. The unique advantage provided by this strain is that the screen is performed at very high concentrations of the antibiotic, non-permissive for most strains. We screened $\sim 4000$ colonies for candidate genes whose disruption led to an increase in norfloxacin susceptibility. The insertion of the transposon affected the transcription of five genes required for the maintenance of resistance in EV18, including acrAB (RND tripartite transporter), waaY (lipopolysaccharide core heptose II kinase), yihO (sulfoquinovose transporter), yhdP (outer membrane permeability factor), and emrK (tripartite efflux pump membrane fusion protein). Three of these genes (YihO, YhdP, and WaaY) have not been previously identified as essential for high-level AMR. Our work provides a new perspective to identify and characterize novel players crucial for maintaining $\mathrm{AMR}$ in E. coli.

\section{RESULTS AND DISCUSSION}

\section{Isolation of E. coli EV18-mutants with increased susceptibility to norfloxacin after Tn5 mutagenesis}

In a previous study, we described the generation of a highly resistant strain to norfloxacin, EV18, by exposing E. coli cells to consecutive increasing concentrations of this antibiotic in an in-vitro evolution experiment (12). In that work, we were able to show the role of multidrug transporters (MDTs) and suppliers on the acquisition of norfloxacinresistance. However, once resistance is achieved, the role of MDTs in maintenance is minor, if at all (12). To understand this phenomenon's complexity, we performed transposon mutagenesis of the EV18 strain to seek for mutants with a decreased resistance to norfloxacin

(Fig. 1A). As a first step, we transformed the EV18 cells with the EZ-Tn5 <KAN-2> Tnp Transposome (Epicentre) to create a library ( 4000 colonies). In the library's initial screening we streaked all the colonies resistant to Kanamycin on LB agar plates containing 100 and 400 $\mu \mathrm{M}$ norfloxacin. Mutants showing an increased susceptibility to norfloxacin were isolated and 
re-tested by growing them in LB liquid medium with norfloxacin at the same concentrations (Fig. 1A). We selected only the mutants that displayed sensitivity to norfloxacin on agar and liquid medium for further transposon screening. In total, 52 candidates completed these requirements. For the mapping, the candidates' genomic DNA was digested to produce bluntended fragments and, consequently, ligated to the anchor bubble unit. All the ligations were further amplified by PCR with specific primers and sequenced. We could map up to 50 out of the 52 transposition events. We then tested plasmid-encoded genes' ability to restore partially or entirely the resistance to norfloxacin and found five that we characterize further, including EV18 Tn5-13 (acrR-acrA), EV18 Tn5-19 (waaY), EV18 Tn5-32 (yihO), EV18 Tn5-47 (yhdP), and EV18 Tn5-48 (emrK). Once the point of insertion was identified, we generated the corresponding nil strains and all the studies described below, except for Tn5-13, were performed in the nil strains.

\section{The non-coding region between acrR and acrA is mutated in EV18 Tn5-13}

The EV18 Tn5-13 mutant sequence analysis revealed that the transposon insertion occurred in the non-coding region between acrR and acrA (Fig. 1B). AcrR regulates the expression of genes involved in multidrug transport (19) and also acts as a global repressor for the mar-sox-rob regulon (20). This mutant's resistance phenotype could be rescued to almost full EV18 levels by simultaneously overexpressing $a c r A$ and $\operatorname{acr} B$ genes but not by the individual genes (Fig. 1C). As depicted in Fig. 1C, the EV18 Tn5-13 strain cannot grow with norfloxacin concentrations higher than $25 \mu \mathrm{M}$. The plasmid-encoded acrB (pSN1 acrB) but not acrA (pT7-7 acrA) confers a small but distinct restoration of resistance. Almost full restoration is observed when both genes $a c r A$ and $a c r B$ are overexpressed together (Fig. 1C). A similar picture was obtained when the mutant and overexpressing strains were grown in LB liquid medium with increasing norfloxacin (Fig. S1).

Although the resistance of EV18 Tn5-13 decreased significantly by at least one order of magnitude it is still more than 10 fold higher than the naïve strain, BW25113. These results confirm our previous analysis of the EV18 $\triangle a c r B$ phenotype where we showed that the medium level resistance is most likely due to the overexpression of other TolC-dependent transporters, mainly AcrF (12).

These results highlight the well-known prominent role of the AcrAB transporter operon in AMR and provide proof of concept for the strategy presented here. 


\section{3. emrK is essential to maintain the high-level resistance of the EV18 strain.}

The EV18 Tn5-48 mutant sequence showed a disruption in the transcription of emrk, coding for a component of the tripartite efflux pump EmrKY-TolC (21), which maps between the emrY and evgL genes (Fig. 2A, Top). We generated an EmrK knockout (EV18 $\Delta e m r K$ ) and, as shown in (Fig. 2B and C) it displays reduced resistance to norfloxacin, with weak growth at $12.5 \mu \mathrm{M}$ norfloxacin. Overexpression of emrK in the EV18 $\Delta e m r K$ strain (EV18 $\Delta e m r K+$ pCA24N emrK) (Fig. 2B) fully restored resistance, indicating that the deletion of emrK solely caused the growth defect. The finding that the gene's deletion in the wild strain does not affect resistance (Fig. 2D) highlights the advantage of using the EV18 strain to reveal genes' function in maintaining resistance. EV18 provides a highly dynamic range of antibiotic concentrations that cannot be reached in the wild type. EmrK role in maintenance of resistance to norfloxacin becomes apparent only at high norfloxacin concentrations.

To explore the scope of EmrK's role in supporting antibiotic resistance, we assayed the growth of EV18 $\Delta$ emrK on agar plates with increasing concentrations of Erythromycin (Ery) and chloramphenicol (Cam) (Fig. S2A). The increased sensitivity of EV18 $\Delta e m r K$ to both antibiotics show that the cross-resistance gained by EV18 to other antibiotics, as norfloxacin and chloramphenicol, is compromised when emrK is deleted. Interestingly, under the conditions tested, the function of emrK appears to be irrelevant in the WT background since its deletion did not generate any change in the phenotype of strain when grown in norfloxacin's presence (Fig. 2D), erythromycin and chloramphenicol (Fig. S2B).

The above-described data show that the expression of emrK in the EV18 strain is required to preserve its resistance also to Erythromycin and Chloramphenicol.

\section{Disruption of waaY transcription weakens the high-level resistance of the EV18 strain.}

The DNA sequence of EV18 Tn5-19 revealed that the transposon insertion affected the waaY gene's transcription. In E. coli, the waaY gene is located in the waa operon, a long transcription unit involved in the lipopolysaccharide (LPS) biosynthesis (Fig. 2A, Bottom). waaY encodes an LPS core heptose (II) kinase that adds a phosphate group to the second heptose residue in the inner core of LPS (22). Changes in the LPS structure can lead to changes in the function and stability of Gram-negative bacteria's outer membrane (23). Growth of EV18 $\Delta$ waaY on agar plates revealed a significant decrease in resistance compared to the 
parent strain, and growth was barely detected at $12.5 \mu \mathrm{M}$ norfloxacin (Fig. 2C). Overexpression of waaY restores norfloxacin's resistance phenotype almost to the EV18 levels: growth is detectable at $100 \mu \mathrm{M}$ norfloxacin (Fig. 2B).

Similar to what we described above for the emrk gene, also the cross resistance of EV18 to Erythromycin and chloramphenicol was compromised in the EV18 $\Delta$ waaY mutant (Fig. S2A). Likewise, under the conditions tested, the function of waaY appears to be irrelevant in the WT background since its deletion did not generate any change in the phenotype of strain when grown in norfloxacin's presence (Fig. 2D), erythromycin and chloramphenicol (Fig. S2B) meaning that the function of waaY is not required for the resistance of the WT cells at the antibiotic concentrations tested.

The results point to an essential link between phosphorylation of LPS and the maintenance of antibiotic resistance in the EV18 strain at the high antibiotic concentration.

\section{Deleting the outer membrane permeability factor encoding gene-yhdP causes a decrease in antibiotic resistance in the EV18 strain.}

Gram-negative bacteria possess a thin peptidoglycan cell wall, surrounded by an outer membrane (OM) containing LPS. The OM is essential for $E$. coli since it acts as a protective barrier against toxic compounds and antibiotics (23). The reduced resistance to norfloxacin of the mutant EV18-Tn5-47 is due to the disruption of the OM permeability factor encoding gene-yhdP. $y h d P$ is located between the metalloprotease subunit $(t / d D)$ and the RNase $G$ (rng)-encoding genes (Fig. 3A, Top). Previous reports indicate that the deletion of yhdP increased the OM's permeability through interaction with the cyclic enterobacterial common antigen (cyclic ECA) (24). Furthermore, $\Delta y h d P$ mutants displayed a decrease in SDS and vancomycin resistance (25) and YhdP is involved in modulating the high-flux phospholipid transport pathway to the outer membrane (26).

As seen in Fig. 4, EV18 $\Delta y h d P$ cells display increased sensitivity to norfloxacin, showing weak growth on agar plates at $25 \mu \mathrm{M}$ norfloxacin and higher. Overexpression of yhdP (pKK yhdP) fully reverts the resistance phenotype (Fig. 4 A). Thus, we concluded that the YhdP activity's disturbance causes the sensitivity of EV18 $\Delta y h d P$ to norfloxacin. Likewise, the cross-resistance of EV18 to norfloxacin and chloramphenicol significantly decreased upon the deletion of $y h d P$ (Fig. 4B). We detected similar results following the growth of these strains in LB-liquid medium with norfloxacin (25-400 $\mu \mathrm{M})$, norfloxacin (25-400 $\mu \mathrm{M})$, and chloramphenicol (0.625- 
$10 \mu \mathrm{M})$ (Fig. S3). The mutation's pleiotropic effect is in accordance with the predicted change in OM structure in this mutant, leading to increased permeability of the antibiotics.

\section{A transporter of the sulfoglycolysis pathway, YihO, is required to maintain the antibiotic resistance in the EV18 strain.}

Sulfoquinovose (SQ; 6-deoxy-6-sulfoglucose) is one of the most abundant compounds in nature and the principal component of plant sulfolipid (27). E. coli K-12 can use SQ as a sole carbon source and energy for growth in the so-called sulfoglycolysis (SFG) pathway (Fig 3B) (28). In this pathway, SQ is transported by the YihO transporter. Afterward, SQ is isomerized to sulfofructose (SF) by the SQ-isomerase YihS. Then, SF is phosphorylated to SF-6-phosphate (SFP), a step catalyzed by the SF-kinase YihV. SFP is subsequently cleaved by the SFP-aldolase (YihT) into sulfolactaldehyde (SLA) and dihydroxyacetone phosphate (DHAP). Finally, SLA is reduced to 2,3-dihydroxypropane-1-sulfonate (DHPS) by the SLA-reductase YihU. Another transporter in the operon, YihP, is 65\% identical in sequence to YihO. It may function to export DHPS but it has not been experimentally characterized. Besides the genes required for SQ transport and catabolism, an additional gene, yihW encoding a transcription factor, is located downstream of the yihV gene. A representation of this operon and the suggested sulfoglycolysis pathway is seen in Fig. 3B. In our work, we identified a mutant (EV18-Tn5-32) whose yihO-transcription was affected by the transposon's insertion in the coding region of this gene. By deleting yihO in the EV18 strain, we noticed a remarkable decrease in antibiotic resistance, not only to norfloxacin (Fig. 4A), but also to erythromycin, and chloramphenicol on agar plates (Fig. 4B). The rescue of the resistance phenotype by overexpressing yihO (pCA24N yihO) confirmed the relevance of yihO in the maintenance of resistance of EV18 (Fig. 4A). As previously described for the emrK and waaY knockouts, the sensitivity of WT $\Delta y$ ihO to the above mentioned antibiotics did not increase (Fig S4).

Since yihO codes for the first protein in the sulfoglycolysis pathway (Fig. 3B), we also constructed the knockout mutants of the genes encoding proteins downstream of yihO in the pathway, a putative 2,3-dihydroxypropane-1-sulfonate export protein (yihP), a 6-deoxy-6sulfofructose-1-phosphate aldolase (yihT), and a 3-sulfolactaldehyde reductase (yihU). The results reveal that the resistance to norfloxacin, erythromycin, and chloramphenicol remains unmodified compared to EV18 (Fig. 5). In other words, EV18 $\Delta y$ ihO was the only mutant from 
the SQ operon (analyzed in this study) whose resistance to norfloxacin, erythromycin, and chloramphenicol underwent a substantial decrease.

yiho codes for a member of the glycoside-pentoside-hexuronide (GPH) cation symporter family of transporters (29). Although its transport mechanism has not been experimentally characterized it has been suggested that it functions as a SQ transporter (28). Since deletion of other genes in the sulfoglycolysis pathway does not have an effect on EV18 resistance we hypothesize that YihO may have other functions essential for resistance.

Therefore, to provide a clue to the possible role of YihO in EV18 resistance, we screened for potential substrates, other than SG. LB is a commonly used nutritionally rich medium for culturing bacteria with a complex composition. To test whether the effect of the deletion of yihO is also observed in media with defined composition, we tested the growth of the WT $\Delta y$ ihO and EV18 $\Delta y$ ihO strains on MMA agar plates with norfloxacin in the presence of different carbon sources, including glucose, glycerol, maltose, and sodium succinate (Fig. S5). In all cases, the growth of EV18 $\Delta y$ ihO was undetectable at $25 \mu \mathrm{M}$ norfloxacin (Fig. S5B). As shown before for the LB medium, under the conditions tested, the deletion of yihO does not render the WT strain more sensitive to norfloxacin (Fig. S5A). We conclude that the effect of the yiho deletion on the phenotype is detected in rich medium as well as in the defined media with various carbon sources (Fig. S5B).

To check whether YihO also works as a drug exporter, we tested the possible impact of YihO overexpression in wild-type cells on resistance to several antibiotics and toxicants but detected no effect (Table S1).

A closer look at the SQ operon's genomic location points to the downstream proximity of a putative outer membrane porin encoded by the gene ompL (Fig. 3B). OmpL localizes to the outer membrane and exhibits porin-type properties allowing solutes smaller than 600 Daltons to pass into and out of the periplasm (30). To rule out the possibility that the deletion of yihO influenced the expression of $o m p L$, we tested the resistance of EV18 $\triangle O m p L$ to different antibiotics (Fig. S6). The growth of EV18 and EV18 $\Delta o m p L$ revealed that ompL deletion does not alter the resistance of EV18 to norfloxacin, norfloxacin, and chloramphenicol (Fig. S6).

We conclude that the loss of resistance of EV18 $\Delta y$ ihO is due to the yihO deletion and rules out a possible positional effect on the expression of ompl. Moreover, the effect of the 
deletion is not due to an alteration in the sulfoglycolysis pathway and further work is required to elucidate the mechanistic basis of this finding.

\section{CONCLUSION}

To fight the increasing numbers of drug-resistant and multidrug-resistant bacteria successfully, we need a detailed knowledge of the molecular mechanisms underlying the acquisition and maintenance of microbial antibiotic resistance.

The approach described in this paper offers an unbiased effort that allows the identification of genes essential for maintaining the very high-level clinically-relevant resistance. Random transposon mutagenesis has been extensively and successfully used prior to this publication. The unique advantage of the work described here is that thanks to the isolation in our lab of the E. coli strain EV18, screening of mutants with decreased resistance were performed at very high concentrations of antibiotics, in our case, norfloxacin.

\section{MATERIALS AND METHODS}

\section{Bacterial strains, growth media, and culture conditions}

Bacterial strains, plasmids and primers used in this study are shown in Tables S2-S4. Escherichia coli strains BW25113 were grown in LB-Na broth $(10 \mathrm{~g} / \mathrm{L}$ tryptone, $5 \mathrm{~g} / \mathrm{L}$ yeast extract and $5 \mathrm{~g} / \mathrm{L} \mathrm{NaCl}$ ), LB-NaPi broth (LB-Na broth with $70 \mathrm{mM}$ sodium phosphate, $\mathrm{pH}=7.4$ ) or minimal medium A (MMA)(31) with $0.5 \%$ glycerol, glucose, maltose or sodium succinate as a carbon source, and supplemented with 1X MEM Amino Acids solutions M550 and M7145. All the antibiotics and reagents used in this study were purchased from Sigma-Aldrich, except for ampicillin (Amp), was from Teva Pharmaceuticals (Petach Tikva, Israel). All the deletion mutants analyzed in this study were created as described by Datsenko and Wanner (32).

\section{Transposon insertion mutagenesis}

EV18 strain was used as the recipient strain for transposon mutagenesis and gene construction. Transposon insertion into E. coli chromosome was carried out by electroporating $1 \mu \mathrm{L}$ of the EZ-Tn5 <KAN-2> Tnp Transposome (Epicentre) in $50 \mu \mathrm{L}$ of EV18pkD46 electrocompetent cells, followed by $2 \mathrm{~h}$ recovery in $1 \mathrm{~mL}$ of SOC medium [0.5\% yeast 
extract, $2 \%$ tryptone, $10 \mathrm{mM} \mathrm{NaCl}, 2.5 \mathrm{mM} \mathrm{KCl}, 10 \mathrm{mM} \mathrm{MgCl}_{2}, 10 \mathrm{mM} \mathrm{MgSO}$, and $20 \mathrm{mM}$ glucose] at $37^{\circ} \mathrm{C}$. Afterwards, the whole transformation was plated on Kan-containing plates $\left(100 \mu \mathrm{L} /\right.$ plate), and incubated overnight at $37^{\circ} \mathrm{C}$. To identify sensitive clones, about 4000 colonies were picked and transferred to LB-norfloxacin plates (0,100 and $400 \mu \mathrm{M})$. To confirm their sensitivity to norfloxacin, colonies that did not grow on 400 and/or $100 \mu \mathrm{M}$ norfloxacin were subjected to another round of analysis in LB liquid medium. Finally, colonies sensitive to norfloxacin on agar and in liquid medium were subjected to transposon mapping.

\section{Transposition mapping}

Transposition mapping was done essentially as described in (33). Genomic DNA was prepared using a NucleoSpin kit (Macherey-Nagel) according to the manufacturers' instructions. Subsequently, gDNA was digested overnight $\left(37^{\circ} \mathrm{C}\right)$ with the restriction enzyme Rsal (New England Biolabs) to produce small, blunt- ended fragments. After digestion, $2 \mu \mathrm{L}$ of anchor bubble unit (see the preparation below), $1 \mu \mathrm{L}$ of $10 \mathrm{mM}$ ATP and $1 \mu \mathrm{L}$ of T4 DNA ligase (New England Biolabs) were added and the ligation reaction was incubated for 5 cycles at $20^{\circ} \mathrm{C}$ for one hour followed by $37^{\circ} \mathrm{C}$ for $30 \mathrm{~min}$. The anchor bubble unit was prepared by annealing the bubble primers (Table S3) in equal amount ( $4 \mu \mathrm{M}$ of each primer) in a total volume of $100 \mu \mathrm{L}$. The mixture was incubated for 5 minutes at $65^{\circ} \mathrm{C}$, and then $\mathrm{MgCl}_{2}$ to a final concentration of 1-2 $\mathrm{mM}$ before cooling down to room temperature. Following ligation, PCR amplification was performed using $2 \mu \mathrm{L}$ of DNA templates (Rsal-digested DNA ligated to the bubbles, as prepared above), $1 \mu \mathrm{L}$ of $20 \mathrm{mM}$ of each primer (KAN-2 FP1 or KAN-2 RP1 complementary to the Tn5 sequence with the bubble primer 224) and $10 \mu \mathrm{L}$ of the 10X Qiagen Multiplex PCR Master Mix Kit (Qiagen, Valencia, CA, USA) in a final volume of $100 \mu \mathrm{L}$. PCR cycling conditions were: $95^{\circ} \mathrm{C}$ for $15 \mathrm{~min}, 35$ cycles of $94^{\circ} \mathrm{C}$ for $30 \mathrm{~s}, 60^{\circ} \mathrm{C}$ for $90 \mathrm{~s}, 72^{\circ} \mathrm{C}$ for $2 \mathrm{~min}$, and a final extension step at $72^{\circ} \mathrm{C}$ for $10 \mathrm{~min}$. The amplified PCR products were separated in $1 \%$ agarose, stained with ethidium bromide and visualized under UV light. DNA bands were excised, purified with the PureLink Qiagen DNA Gel Extraction Kit and sent out for sequencing. DNA sequencing analysis was carried out using specific primers to the $\operatorname{Tn} 5$ and bubble regions. DNA sequences were analyzed by BLAST and compared to the EV18 sequence in order to find the accurate insertion place of the transposon. 


\section{Susceptibility assays to antibiotics of $E$. coli strains}

Pre-cultures were grown overnight in $4 \mathrm{~mL}$ of LB-Na and diluted 1:100 in either LB-Na or MMA $(\mathrm{pH}=7.4)$. Cells continued growing for several hours (until early logarithmic phase) and diluted to a final $\mathrm{OD}_{600}=0.02$ in either LB-NaPi or MMA for examining their growth in liquid media. Duplicate two fold serial dilutions were made in 96-well for each antibiotic, and one well only with medium in order to rule out contamination. Growth was followed for $16 \mathrm{~h}$, measuring the optical density at $600 \mathrm{~nm}$ every hour, at $37^{\circ} \mathrm{C}$ and constant shaking in a Synergy 2 Microplate Reader (Bio Tek). Results were obtained by comparing the percentage of growth of the tested strain grown without antibiotics with the strain grown in the presence of them. Growth curves were obtained by using the GraphPad Prism 7 software. For the phenotypes on solid medium, the cells were grown as indicated above but instead of diluting them to an $\mathrm{OD}_{600}=0.02$, they were brought to $\mathrm{OD}_{600}=0.2$ and $5 \mu \mathrm{L}$ of 10 -fold serial dilutions of the cells were spotted on LB-NaPi $(\mathrm{pH}=7.4)$ agar plates containing increasing concentrations of antibiotics according to the experimental setup. Plates were then incubated $\left(37^{\circ} \mathrm{C}\right.$, overnight), and growth of the strains was recorded by using the Fusion Fx ECL and Gel Documentation System (VILBER). Biological replicates were included for each experiment.

\section{Sofware}

The processing of the graphs was performed with GraphPad Prism 7 software. The images describing the methodology for the transposon mutagenesis (Fig. 1), as well as in Fig. 2 and 3 shown in this work were created with BioRender.

Funding Information: This work was supported by Grant 143/16 from the Israel Science Foundation and Grants A1004 and M497-F1 from the Rosetrees Trust. The funders had no role in study design, data collection, and interpretation, or the decision to submit the work for publication

Acknowledgment: SS is Mathilda Marks-Kennedy Emeritus Professor of Biochemistry at the Hebrew University of Jerusalem. The authors declare non-competing interests. 


\section{References}

1. K. Bush et al., Tackling antibiotic resistance. Nature reviews 9, 894-896 (2011).

2. J. Carlet et al., Society's failure to protect a precious resource: antibiotics. Lancet 378, 369-371 (2011).

3. C. f. D. Control., Antibiotic resistance threats in the United States, 2019. (2019).

4. W. H. O. WHO World Health Organization, Antimicrobial Resistance Global Report on surveillance. (2014).

5. B. P. Alcock et al., CARD 2020: antibiotic resistome surveillance with the comprehensive antibiotic resistance database.

6. I. Yelin, R. Kishony, Antibiotic Resistance. Cell 172, 1136-1136.e1131 (2018).

7. D. Hughes, D. I. Andersson, Evolutionary Trajectories to Antibiotic Resistance. Annu Rev Microbiol 71, 579-596 (2017).

8. X. Z. Li, P. Plesiat, H. Nikaido, The challenge of efflux-mediated antibiotic resistance in Gram-negative bacteria. Clinical microbiology reviews 28, 337-418 (2015).

9. J. M. Blair, M. A. Webber, A. J. Baylay, D. O. Ogbolu, L. J. Piddock, Molecular mechanisms of antibiotic resistance. Nature reviews 13, 42-51 (2015).

10. R. Laxminarayan et al., Antibiotic resistance-the need for global solutions. The Lancet infectious diseases 13, 1057-1098 (2013).

11. G. D. Wright, Molecular mechanisms of antibiotic resistance. Chem Commun (Camb) 47, 4055-4061 (2011).

12. Y. Shuster, S. Steiner-Mordoch, N. Alon Cudkowicz, S. Schuldiner, A Transporter Interactome Is Essential for the Acquisition of Antimicrobial Resistance to Antibiotics. PLoS One 11, e0152917 (2016).

13. N. Judson, J. J. Mekalanos, Transposon-based approaches to identify essential bacterial genes. Trends Microbiol 8, 521-526 (2000).

14. T. van Opijnen, A. Camilli, Transposon insertion sequencing: a new tool for systemslevel analysis of microorganisms. Nature reviews 11, 435-442 (2013).

15. T. C. Meredith, H. Wang, P. Beaulieu, A. Gründling, T. Roemer, Harnessing the power of transposon mutagenesis for antibacterial target identification and evaluation. Mob Genet Elements 2, 171-178 (2012).

16. W. S. Reznikoff, K. M. Winterberg, Transposon-based strategies for the identification of essential bacterial genes. Methods Mol Biol 416, 13-26 (2008).

17. N. T. Liberati et al., An ordered, nonredundant library of \&lt;em\&gt;Pseudomonas aeruginosa\&lt;/em\&gt; strain PA14 transposon insertion mutants. Proceedings of the National Academy of Sciences of the United States of America 103, 2833 (2006).

18. M. S. Sonnabend et al., Identification of Drug Resistance Determinants in a Clinical Isolate of \&lt;span class=\&quot;named-content genus-species\&quot; id=\&quot;named-content-1\&quot;\&gt;Pseudomonas aeruginosa\&lt;/span\&gt; by High-Density Transposon Mutagenesis. Antimicrobial Agents and Chemotherapy 64, e01771-01719 (2020).

19. D. Ma, M. Alberti, C. Lynch, H. Nikaido, J. E. Hearst, The local repressor AcrR plays a modulating role in the regulation of acrAB genes of Escherichia coli by global stress signals. Mol Microbiol 19, 101-112 (1996).

20. J. O. Lee, K. S. Cho, O. B. Kim, Overproduction of AcrR increases organic solvent tolerance mediated by modulation of SoxS regulon in Escherichia coli. Appl Microbiol Biotechnol 98, 8763-8773 (2014). 
21. H. Tanabe et al., Growth phase-dependent transcription of emrKY, a homolog of multidrug efflux emrAB genes of Escherichia coli, is induced by tetracycline. The Journal of general and applied microbiology 43, 257-263 (1997).

22. J. A. Yethon, D. E. Heinrichs, M. A. Monteiro, M. B. Perry, C. Whitfield, Involvement of waaY, waaQ, and waaP in the modification of Escherichia coli lipopolysaccharide and their role in the formation of a stable outer membrane. J Biol Chem 273, 2631026316 (1998).

23. T. J. Silhavy, D. Kahne, S. Walker, The bacterial cell envelope. Cold Spring Harb Perspect Biol 2, a000414 (2010).

24. A. M. Mitchell, T. Srikumar, T. J. Silhavy, Cyclic Enterobacterial Common Antigen Maintains the Outer Membrane Permeability Barrier of \&lt;span class=\&quot;named-content genus-species\&quot; id=\&quot;named-content1\&quot;\&gt;Escherichia coli\&lt;/span\&gt; in a Manner Controlled by YhdP. MBio 9, e01321-01318 (2018).

25. A. M. Mitchell, W. Wang, T. J. Silhavy, Novel RpoS-Dependent Mechanisms Strengthen the Envelope Permeability Barrier during Stationary Phase. J Bacteriol 199 (2017).

26. J. Grimm et al., The inner membrane protein YhdP modulates the rate of anterograde phospholipid flow in \&lt;em\&gt;Escherichia coli\&lt;/em\&gt. Proceedings of the National Academy of Sciences 10.1073/pnas.2015556117, 202015556 (2020).

27. J. L. Harwood, R. G. Nicholls, The plant sulpholipid-- a major component of the sulphur cycle. Biochem Soc Trans 7, 440-447 (1979).

28. K. Denger et al., Sulphoglycolysis in Escherichia coli K-12 closes a gap in the biogeochemical sulphur cycle. Nature 507, 114-117 (2014).

29. B. Poolman et al., Cation and sugar selectivity determinants in a novel family of transport proteins. Molecular Microbiology 19, 911-922 (1996).

30. C. Dartigalongue, H. Nikaido, S. Raina, Protein folding in the periplasm in the absence of primary oxidant DsbA: modulation of redox potential in periplasmic space via OmpL porin. EMBO J 19, 5980-5988 (2000).

31. B. Davies, E. Mingioli, Mutants of Escherichia coli requiring methionine or vitamin B12. J Bacteriol 60, 17-28 (1950).

32. K. A. Datsenko, B. L. Wanner, One-step inactivation of chromosomal genes in Escherichia coli K-12 using PCR products. Proc Natl Acad Sci U S A 97, 6640-6645 (2000).

33. T. Ducey, D. Dyer (Rapid Identification of EZ : : TN TM Transposon Insertion Sites in the Genome of Neisseria gonorrhoeae. 
Figures

$\mathbf{A}$

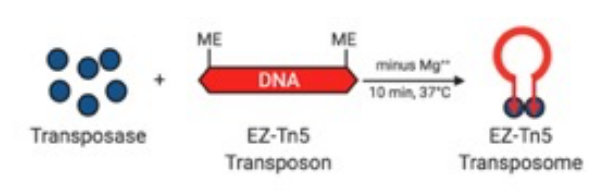

Fig.1

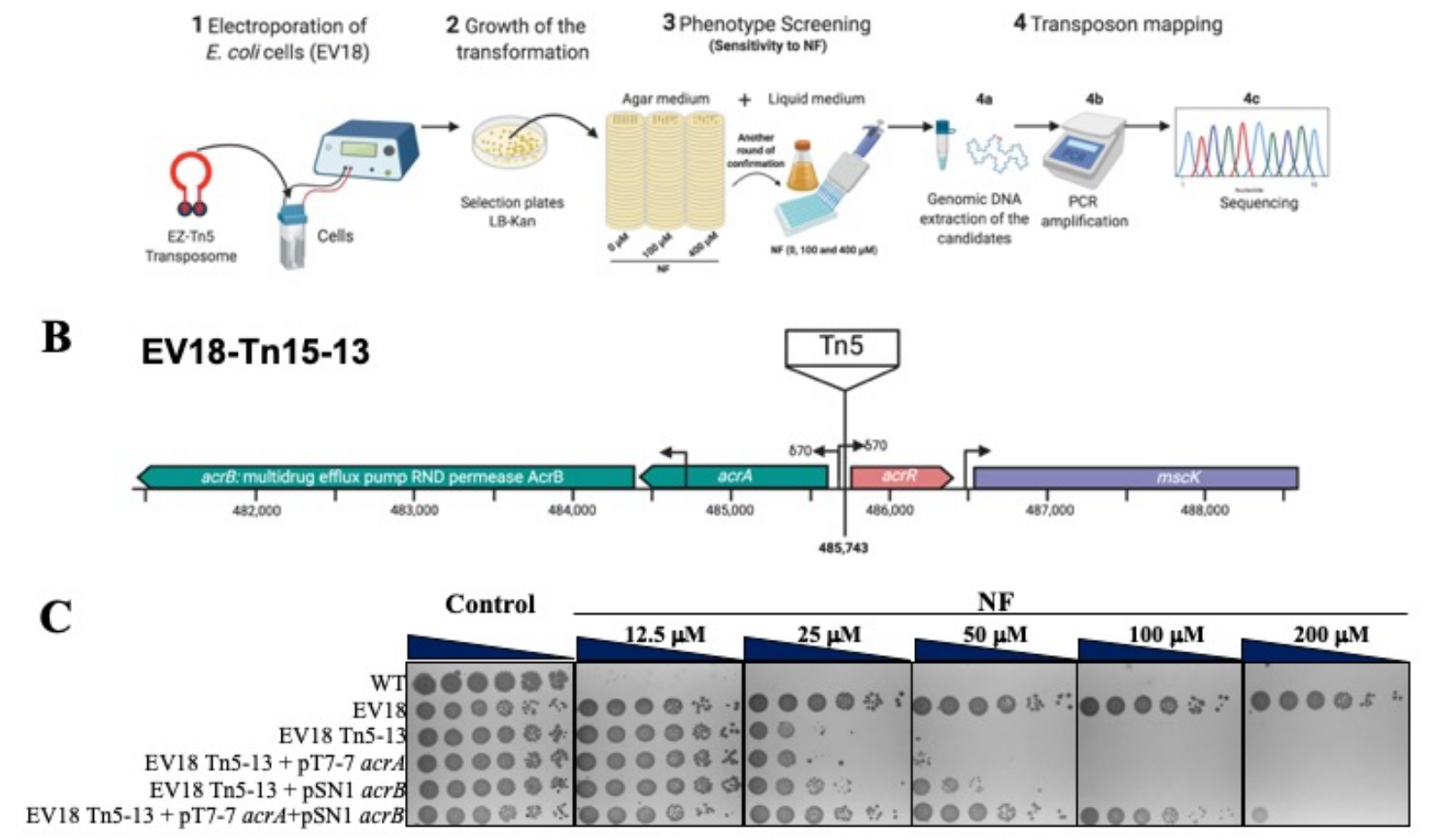

Fig. 1. Transposon mutagenesis for identifying candidate genes required for the maintenance of the NF-resistance in the EV18 strain. (A) Generation of the transposon library in the NF-resistant E. coli strain EV18. Top: Formation of the transposon complex EZ$\operatorname{Tn} 5$ by incubating the EZ-Tn5 transposase enzyme with the EZ-Tn 5 transposon, containing the Tn903 kanamycin resistance gene $\left(\mathrm{Kan}^{\mathrm{R}}\right)$ flanked by the Mosaic End (ME) transposase recognition sequences. Bottom: Steps describing the process of selecting EV18- transposition mutants with increased sensitivity to NF. (1) Electroporation of the EV18 cells with the EZ-Tn5 transposome. After recovery of the transformed cells, (2) cells were spread onto LB-Kan agar plates and incubated overnight at $\left(37^{\circ} \mathrm{C}\right)$. Each colony $(\sim 4000)$ was picked and streaked on LB-agar plates containing 100 and $400 \mu \mathrm{M}$ NF to screen for this antibiotic's sensitivity (3). The growth of selected candidates in LB medium with NF confirmed their sensitivity, which was studied further. (4) The specific Tn5 insertion sites of these mutants were identified by DNA sequencing (for more details, see Materials and Methods). (B) Location of the acrA and acrR 
genes in the genomic context of E. coli (C) The sensitivity of the transposon mutant EV18 Tn513 to NF. Growth of the EV18-Tn5-13 mutant on LB-agar plates (+/- NF, at $37^{\circ} \mathrm{C}$ ) with or without the complementation plasmids for overexpressing either acrA (pT7-7 acrA), acrB (pSN1 $a c r B$ ) or both. Major restoration of resistance is achieved when acrA and acrB are simultaneously overexpressed.

$\mathbf{A}$

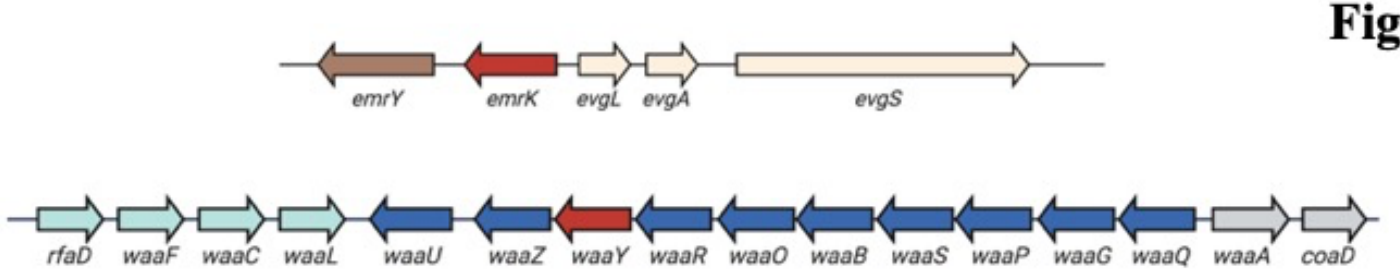

B

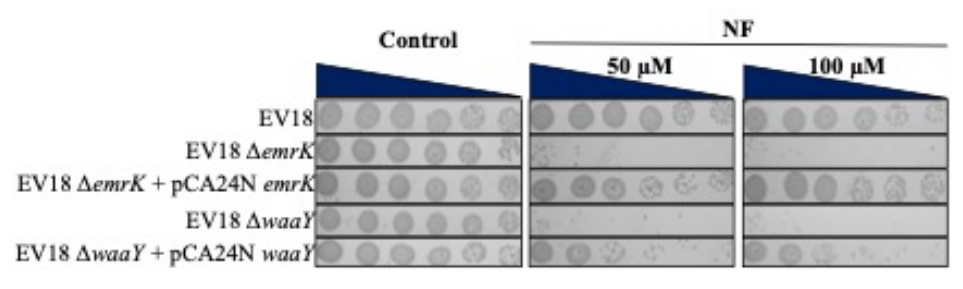

C

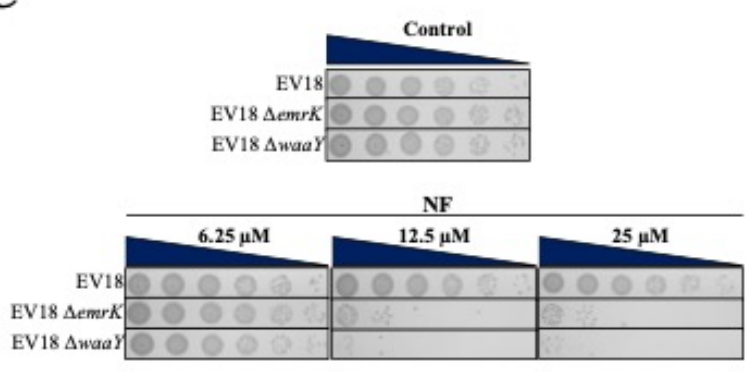

D

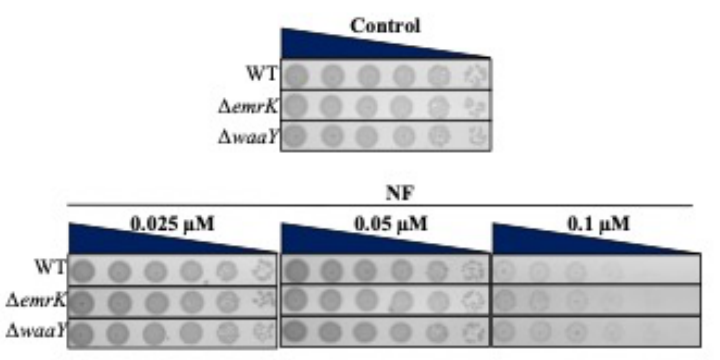

Fig. 2. emrK and waaY are required to maintain the high-level resistance of EV18 to NF (A) Location of the emrK and waaY genes in the genomic context of E. coli. Top: The emrK gene, encoding the membrane fusion protein of a tripartite efflux pump complex, is flanked by emrY and evgL, encoding a subunit of the tripartite efflux pump membrane and a small unannotated protein, respectively. Bottom: The waa locus (formerly rfa) contains the three major gene operons for lipopolysaccharides (LPS) biosynthesis in E. coli. The operons are identified by the first gene of the transcriptional unit as $r f a D$ (green), waaQ (blue), and waaA (gray). The waaY gene, encoding a lipopolysaccharide core heptose (II) kinase, belongs to the waaQ operon, which encodes enzymes involved in the core oligosaccharide (OS) assembly. 
Genes are represented as arrows. (B-C) Phenotypes of the $\Delta e m r K$ and $\Delta$ waa $Y$ strains grown on LB-agar plates in the presence of increasing concentrations of norfloxacin (NF), as indicated on top of the plates. Deletion mutants generated in the background of the EV18 strain are shown in B and C, while the mutants in the WT (BW25113) are in D. The growth of the EV18 $\Delta e m r K$ and EV18 $\Delta$ waaY strains on LB plates with NF was restored almost to the EV18 levels after transformation of the deletion mutants with the plasmids pCA24N emrK and pCA24N waaY respectively.

$\mathbf{A}$

Fig.3

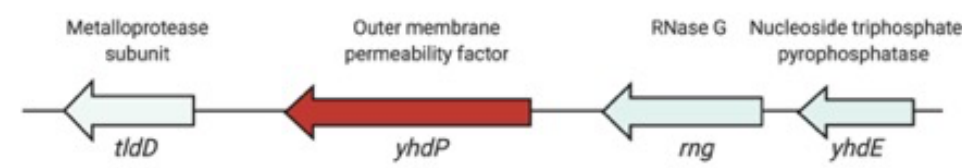

B

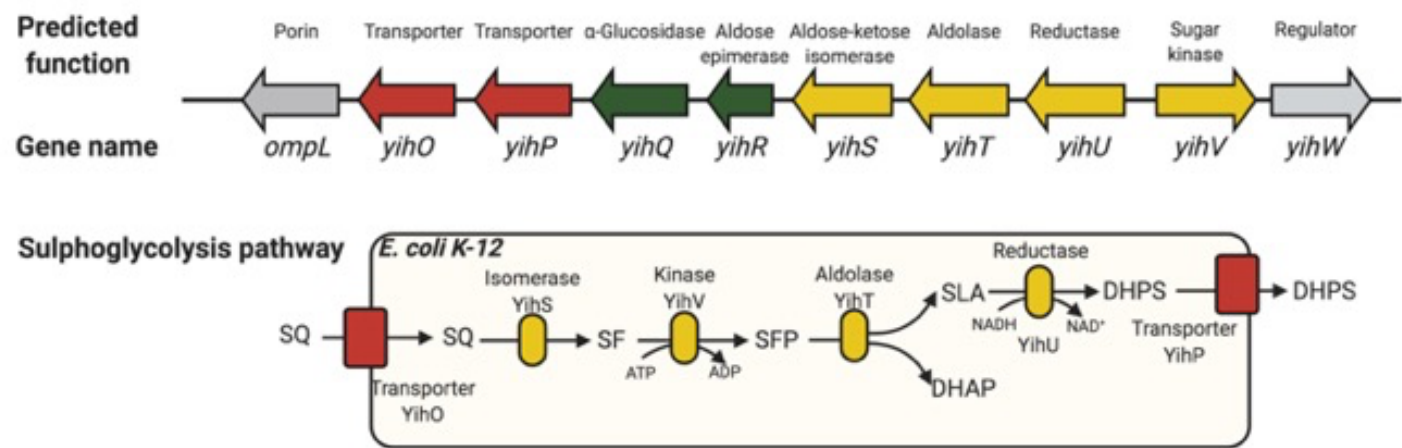

Fig. 3. Genomic context of the yhdP and yihO genes in E. coli. (A) The $y$ hdP gene (in red) encodes for a large protein implicated in maintaining outer membrane permeability through its functional interaction with cyclic enterobacterial common antigen (cyclic ECA). (B) Representation of the operon implicated in the sulfoglycolysis pathway. The yihW gene product controls the transcription of the operon. (Bottom) Predicted pathway for the catabolism and transport of sulfoquinovose (SQ) (adapted from [Denger, 2014 \#8209]). Abbreviations: SQ, sulfoquinovose; SF, 6-deoxy-6-sulfofructose; SFP, 6-deoxy-6- 
sulfofructose-1-phosphate; SLA, 3-sulfoacetaldehyde; DHAP, dihydroxyacetone phosphate; DHPS, 2,3-dihydroxypropane sulfonate.

$\mathbf{A}$

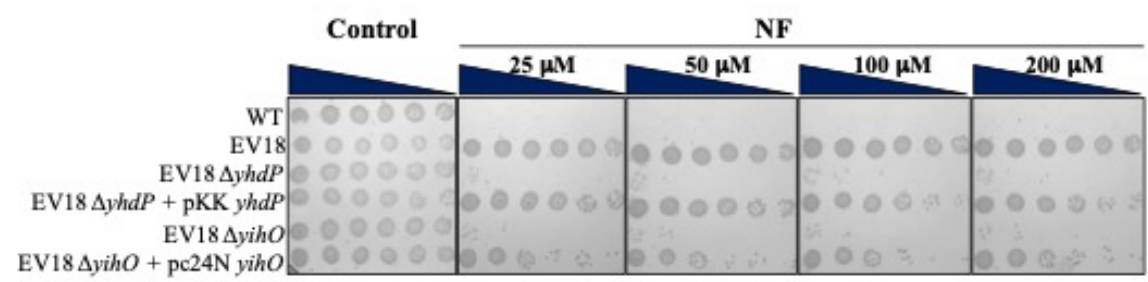

B

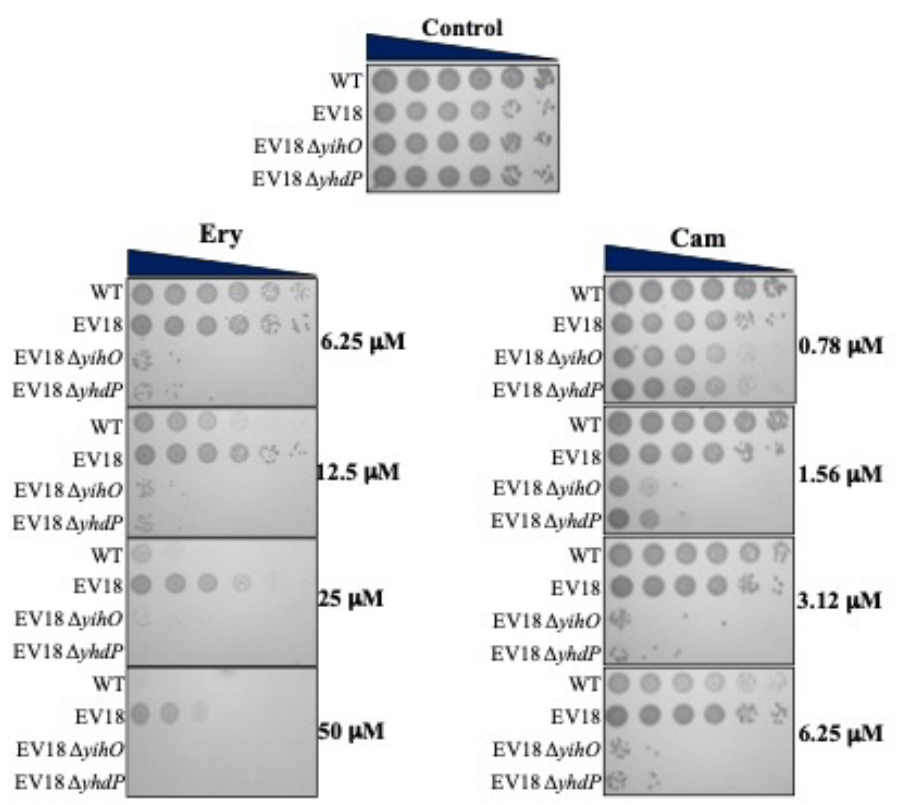

Fig. 4. YihO and $y$ hdP are required to maintain the high-level resistance of EV18 to NF, Ery, and Cam. (A) The deletion of yihO and yhdP reduces the resistance of the EV18 strain to NF. Quantitative restoration of the resistance is achieved in cells bearing plasmids $\mathrm{pC} 24 \mathrm{~N}$ yihO and pKK yhdP, respectively. Concentrations of NF are indicated on top of each plate. All plates included $50 \mu \mathrm{M}$ IPTG. (B) The deletion of yihO and $y h d P$ also reduces the resistance of the EV18 strain to Ery and Cam (left and right, respectively). Concentrations for each antibiotic are indicated on the right side of the plates. 

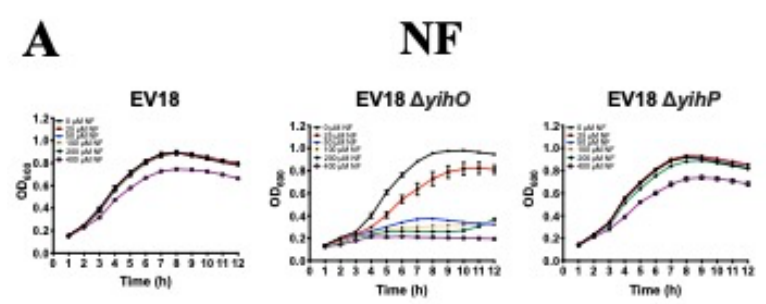

B

Ery

Fig.5

EV18 $\Delta$ yihT

EV18 $\Delta$ yihU
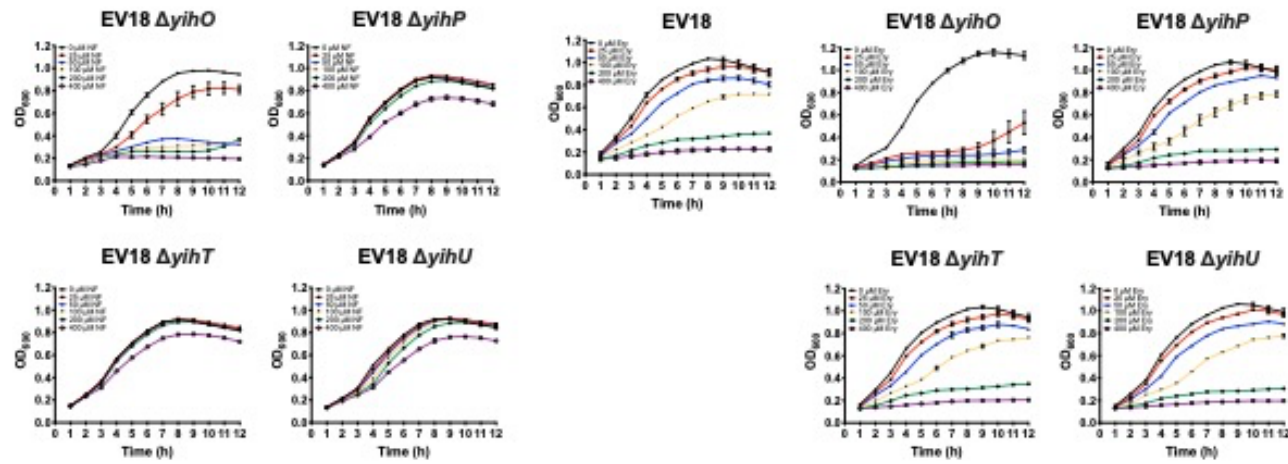

EV18 $\Delta$ yihT

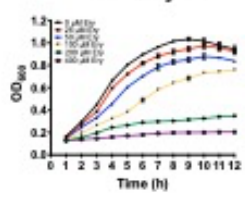

EV18 $\Delta$ yihU

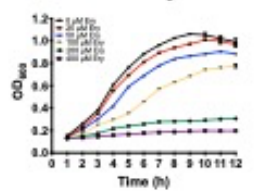

C

Cam
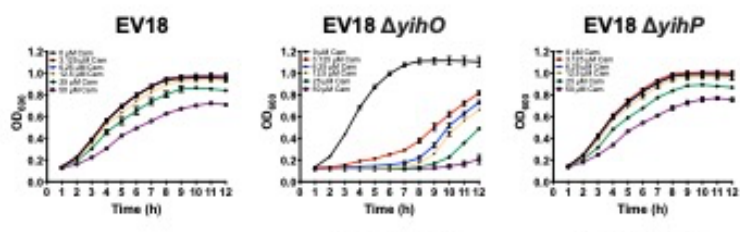

EV18 $\Delta y$ ihT

EV18 $\Delta y$ ihu
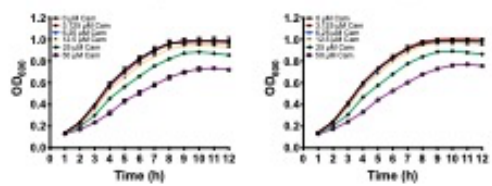

Fig. 5. The deletion of yiho but not of other genes in the operon affects the resistance of the EV18 strain to several antibiotics. Liquid cultures of EV18, EV18 $\Delta y i h O$, EV18 $\Delta y i h P$, EV18 $\Delta y i h T$, and $\mathrm{EV} 18 \Delta y i h U$, were prepared in LB medium to an $\mathrm{OD}_{600}=0.01$ and transferred to a microtiter plate with the indicated concentrations of antibiotics. The growth of the cells was recorded every hour by a microtiter plate reader. The antibiotics used in this study were $(\mathbf{A})$ NF, (B) Ery, and (C) Cam. From all the deletion mutants, only EV18 $\Delta y$ ihO showed an effect on growth with all the tested antibiotics. 